\title{
A Hybrid EKF and Switching PSO Algorithm for Joint State and Parameter Estimation of Lateral Flow Immunoassay Models
}

\author{
Nianyin Zeng, Zidong Wang, Yurong Li, Min Du and Xiaohui Liu
}

\begin{abstract}
In this paper, a hybrid extended Kalman filter (EKF) and switching particle swarm optimization (SPSO) algorithm is proposed for jointly estimating both the parameters and states of the lateral flow immunoassay model through available short time-series measurement. Our proposed method generalizes the well-known EKF algorithm by imposing physical constraints on the system states. Note that the state constraints are encountered very often in practice that give rise to considerable difficulties in system analysis and design. The main purpose of this paper is to handle the dynamic modeling problem with state constraints by combining the extended Kalman filtering and constrained optimization algorithms via the maximization probability method. More specifically, a recently developed SPSO algorithm is used to cope with the constrained optimization problem by converting it into an unconstrained optimization one through adding a penalty term to the objective function. The proposed algorithm is then employed to simultaneously identify the parameters and states of a lateral flow immunoassay model. It is shown that the proposed algorithm gives much improved performance over the traditional EKF method.
\end{abstract}

Index Terms-lateral flow immunoassay; extended Kalman filtering; switching particle swarm optimization; constrained optimization; parameter estimation.

\section{INTRODUCTION}

Copyright (c) 2011 IEEE. Personal use of this material is permitted. However, permission to use this material for any other purposes must be obtained from the IEEE by sending a request to pubs-permissions@ieee.org.

N. Zeng and Y. Li are with the College of Electrical Engineering and Automation, Fuzhou University, Fuzhou 350002, P. R. China, and also with the Fujian Key Laboratory of Medical Instrumentation and Pharmaceutical Technology, Fuzhou 350002, P. R. China. E-mail: nianyin.zeng@gmail.com, liyurong@fzu.edu.cn.

Z. Wang and X. Liu are with the Department of Information Systems and Computing, Brunel University, Uxbridge, Middlesex, UB8 3PH, United Kingdom. E-mail: Zidong.Wang@ brunel.ac.uk, Xiaohui.Liu@brunel.ac.uk.

M. Du is with the Fujian Key Laboratory of Medical Instrumentation and Pharmaceutical Technology, Fuzhou University, Fuzhou 350002, P. R. China. E-mail: dm_dj90@163.com.
$\mathbf{T}$ HE rapid immunochromatographic test strip, also called lateral flow immunoassay (LFIA), has been extensively investigated and widely used in many fields over the past decades owing to its attractive properties such as short analysis time, ease of use, low cost, high sensitivity, good specificity and satisfactory stability [26], [43]. Recently, researchers have been focusing on not only the improvement of the biochemical properties of the strips via material selection (see e.g. [7], [11], [16], [34]) but also the development of the quantitative instruments (see e.g. [5], [6], [8], [17], [18]). On the other hand, in order to produce strips with high-sensitivity and low constant of variance for the purpose of quantification, there has been a growing research interest in establishing a mathematical model that allows us to predict kinetic characteristics and test the effects of various design parameters in a both rapid and inexpensive way. In addition to providing insights into device operation, such a model could also enable us to optimize device performance [23], [24], [45].

In [23], [24], the convection diffusion reaction equations have been used to model the lateral flow immunoassay systems and the simulation has been carried out by using the COMSOL software. Very recently, in [45], a nonlinear state-space model for sandwich-type lateral flow immunoassay has been developed via the extend Kalman filter (EKF) algorithm. Because of its versatility and effectiveness, the EKF algorithm is capable of overcoming some difficulties in system modeling brought from short time-series data, nonlinearities and incomplete measurement. Moreover, EKF algorithm performed like the method used in [38]-[40] is well known for its ability in estimating the system parameters and system states simultaneously. Essentially, the EKF assumes that there is no constraint on the value of the system states, i.e., the system states are allowed to be positive, negative or zero. Unfortunately, this is not true for many practical models. For example, in 
the lateral flow immunoassay model, the concentration of the materials, the association and dissociation rate constants should be positive in the real world. Incorporating constraints (algebraic equality and/or inequality) directly on the system states in EKF is a non-trivial task because, mathematically, the recursive nature of the EKF algorithm will no longer be valid with the state constraints. As such, there is a great need to seek an alternative approach for tackling the so-called constrained estimation issue that aims to maintain the nice properties of EKF algorithm while enforcing the state constraints in the EKF framework. In search of such an approach, the maximum probability method [29], [30] appears to be an ideal candidate for converting the constrained estimation problem to constrained optimization after each time step of the EKF algorithm.

On another research forefront, the constrained optimization problems have recently gain considerable research attention since they are frequently encountered in many applications such as engineering design, very large scale integration design, structural optimization, economics, locations and allocation problems [22]. In particular, the Particle Swarm Optimization (PSO) is a global evolutionary algorithm developed by Kennedy and Eberhart [12] that stimulates the social behaviors of birds blocking or fish schooling, etc. The PSO algorithm has been successfully applied in a variety of fields especially for unconstrained optimization problems due to its effectiveness in performing difficult optimization tasks and its convenience for implementation with fast convergence to a reasonably good solution [10], [12], [20], [27], [28], [35], [37]. Up to now, several variants of PSO have been proposed in [10], [20], [27], [28], [35]. For example, in [35], a switching PSO algorithm has been developed that introduces a mode-dependent velocity updating equation with Markovian switching parameters in order to overcome the contradiction between the local search and global search. The switching PSO algorithm developed in [35] can not only avoid the local search stagnating in a local area and wasting more time on a invalid search but also lead the swarm move to a more potential area quickly, which helps to obtain a global search greatly. Although the PSO algorithm has been developed primarily as the unconstrained optimization method, it performs well when used in constrained optimization problems [22]. It is worth mentioning that the penalty function approach associated PSO algorithm has been the most popular constraint-handling technique because of its simple principle for converting a constrained optimization problem to an unconstrained optimization one by adding a penalty term to the objective function [22], [32]. Inspired by the above discussion, in this paper, we propose to use a nonstationary multi-stage assignment penalty function ( [22]) to further improve the switching PSO algorithm in order to deal with the constrained EKF problem for joint state and parameter estimation of lateral flow immunoassay models.

In this paper, we aim to develop a hybrid EKF and switching PSO algorithm for jointly estimating system parameters and states of the lateral flow immunoassay model through available short time-series measurement. The identified lateral flow immunoassay model is proven to be more accurate than the one obtained from the traditional EKF algorithm. The main contribution of this paper is mainly threefold. 1) A hybrid EKF and switching PSO algorithm is proposed to jointly estimate the states and parameters in a simultaneous way, thereby generalizing the well-known EKF algorithm with imposed physical constraints on the system states. 2) The developed algorithm is applied to model the lateral flow immunoassay system, which represents the first of few attempts of the kind. It is shown that the system parameters, actual concentration distribution of the states, the system noise and measurement noise in the nonlinear model of lateral flow immunoassay can all be identified simultaneously through iterative procedure by using a small number of observations. 3) Comparisons show that the proposed algorithm provides much improved performance over the unconstrained extended Kalman filtering.

The rest of this paper is organized as follows. The lateral flow immunoassay model is introduced in Section II. In Section III, EKF algorithm with inequality constraints on the states is introduced by incorporating the maximum probability method. In Section IV, the switching PSO algorithm for constrained optimization problems is described. The results of joint parameter and state estimation by the hybrid EKF and switching PSO algorithm are discussed in Section V. Finally, concluding remarks are given in Section VI.

\section{The Lateral Flow Immunoassay Model And} PROBLEM FORMULATION

A typical configuration of lateral flow immunoassay, as shown in Fig. 1 [3], consists of a variety of materials such as sample pad, nitrocellulose membrane, conjugate pad and wicking pad. The primary antibodies are immobilized within a defined detection zone (test line) on the membrane. The secondary antibodies are conjugated with reporter particles such 
as colloidal gold, carbon black, fluorescent, or paramagnetic monodisperse latex particle [1]. In this paper, we focus on the sandwich format of gold immunochromatographic strip where the reporter particle uses the colloidal gold nanoparticles. With the presence of an antigen in the sample, a sandwich-type assay is formed between the secondary antibody-immobilized gold nanoparticle immunocomplex and the primary antibody immobilized on the membrane. After the antigen-antibody reaction, the red color caused by the accumulation of gold nanoparticle at that location would appear on the membrane [1], [26]. The color intensity of the red test line (signal intensity), which relates directly to the concentration of the target protein in the standard or spiked samples, is assessed visually or by a reader system for quantitative analysis [34].

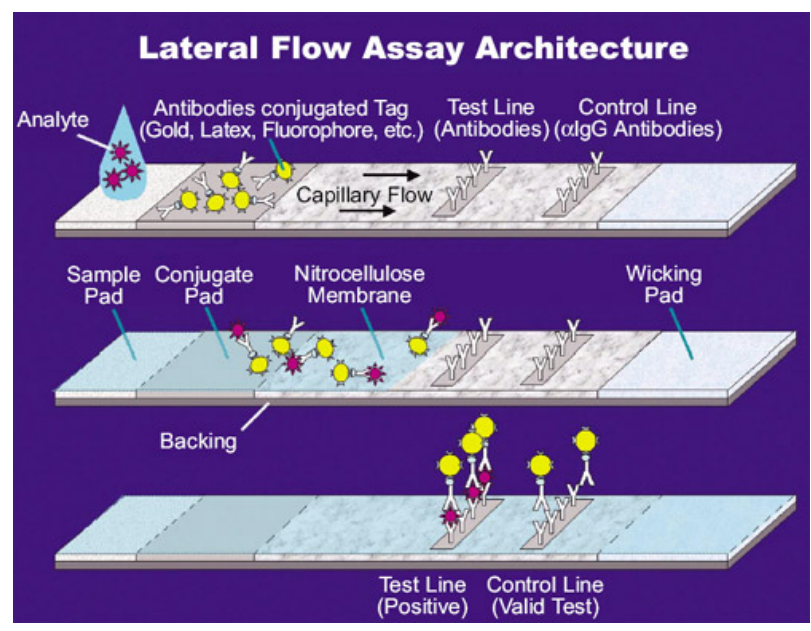

Fig. 1. Lateral flow immunoassay architecture.

In general, the biochemical reactions of the lateral flow immunoassay signal pathway without consideration of the control line can be summarized as follows [23]:

1) Assume that the sample contains various target analytes $A_{i}$. When the sample migrates through the conjugate pad, the analytes interact with the particulate color particle conjugate $P$ to form particle-analyte complexes $P A_{i}$,

$$
A_{i}+P \underset{k_{2}}{\stackrel{k_{1}}{\rightleftharpoons}} P A_{i}
$$

2) The free analytes in the sample and the particle-analyte complexes both migrate into the membrane by the capillary action. Free analytes of type $i\left(A_{i}\right)$ and particleanalyte complexes $P A_{i}$ interact with the immobilized ligands of type $i\left(R_{i}\right)$ to form the complexes,

$$
A_{i}+R_{i} \underset{k_{4}}{\stackrel{k_{3}}{\rightleftharpoons}} R A_{i}
$$

$$
P A_{i}+R_{i} \underset{k_{6}}{\stackrel{k_{5}}{\rightleftharpoons}} R P A_{i}
$$

3) Additionally, unbound particulate conjugate $P$ may bind to the complex $R A_{i}$ to form the complex $R P A_{i}$,

$$
P+R A_{i} \underset{k_{8}}{\stackrel{k_{7}}{\rightleftharpoons}} R P A_{i}
$$

In this paper, for simplicity, we only consider a single target analyte in the sample, therefore we drop the subscript $i$ from the next section. Let $x_{1}, x_{2}, x_{3}, x_{4}, x_{5}$ and $x_{6}$ be the concentration of $A, P, P A, R, R A$ and $R P A$, respectively. For demonstration purpose, it is assumed that there is no timedelay between the biochemical reactions (1)-(4). The rates of the reactions are defined as follows:

$$
\begin{aligned}
& v_{1}=k_{1} x_{1} x_{2}-k_{2} x_{3} \\
& v_{2}=k_{3} x_{1} x_{4}-k_{4} x_{5} \\
& v_{3}=k_{5} x_{3} x_{4}-k_{6} x_{6} \\
& v_{4}=k_{7} x_{2} x_{5}-k_{8} x_{6}
\end{aligned}
$$

where $k_{1}, k_{3}, k_{5}, k_{7}$ and $k_{2}, k_{4}, k_{6}, k_{8}$ are the association and dissociation rate constants, respectively. The stoichiometrix for the biochemical reaction of the lateral flow immunoassay is given by

$$
S=\left[\begin{array}{cccc}
-1 & -1 & 0 & 0 \\
-1 & 0 & 0 & -1 \\
1 & 0 & -1 & 0 \\
0 & -1 & -1 & 0 \\
0 & 1 & 0 & -1 \\
0 & 0 & 1 & 1
\end{array}\right]
$$

Let $x=\left[x_{1}, x_{2}, \ldots, x_{6}\right]^{T}$ and $V=\left[v_{1}, v_{2}, \ldots, v_{4}\right]^{T}$. The differential equation for the biochemical reactions of the lateral flow immunoassay is given as follows [31], [33]

$$
\frac{d x(t)}{d t}=S V(x)
$$

It should be pointed out that the variables $x_{1}, x_{2}, x_{3}, x_{4}, x_{5}$ and $x_{6}$ are not measurable/observable. The only observed signal that can be detected with a reader system is the test line's intensity, which is typically either the color intensity or the phosphor emission intensity or fluorescent [23]. The signal would be proportional to the concentration of particleanalyte complexes $P A$ and the complex $R P A$. In this case, the observation equation is obtained as follows

$$
y=k_{9}\left(x_{3}+x_{6}\right)
$$

The most general form of the nonlinear model for the dynamics of biochemical networks is defined by dynamic mass 
balance equations or kinetic models [33], where the system consists of a pair of equations as follows

$$
\begin{aligned}
\frac{d x}{d t} & =S V(x(t))+G(t) w(t) \\
z(t) & =g(x(t))+L(t) v(t)
\end{aligned}
$$

where $x(t)$ is the vector of state variables which are concentrations of antibodies, antigens or complex material; $y(t)$ is the measurement process; $S V(x(t))$ with $S$ being a stoichiometric matrix that describes the biochemical transformation in a biochemical network and $V(x(t))$ being the vector of reaction rates (usually the vector of nonlinear function of the state) [33]; $G(t)$ and $L(t)$ are arbitrary time-varying matrices independent of $x(t)$ and $y(t) ; g(x(t))$ is the measurement model function; $w(t)$ and $v(t)$ are system noise and measurement noise, respectively. The system noise appearing in (11) is determined by the structure, reaction rates, and concentrations of antibodies, antigens or complex material. The system noise typically results from the transmitted fluctuations of each of the materials in the biochemical network or fluctuations in the rates of the basic reactions that affect the state variables. On the other hand, the measurement noise in (12) is unavoidable in biochemical systems that might come from the sensor inaccuracy and environment changes (e.g. light variation). Due to the random nature as well as the different sources for the system and measurement noises, it makes sure to assume that these two kinds of noises are zero-mean uncorrelated Gaussian noises.

In practice, when modeling biochemical networks from observed data (time series), discrete-time models play a more crucial role than their continuous-time counterparts in today's digital world. In order to obtain the nonlinear model for lateral flow immunoassay biochemical networks from discretely obtained measurements, it is usually essential to formulate the discrete-time analogue as follows [33]:

$$
\begin{aligned}
x(k+1) & =x(k)+S V(x(k))+w(k) \\
z(k) & =g(x(k))+v(k)
\end{aligned}
$$

To facilitate the parameter estimation, in this paper, let us use $\theta=\left[k_{1}, k_{2}, \ldots, k_{9}\right]^{T}$ to denote the parameters to be estimated, which are the association and dissociation rate constants in the vector $V(x(k))$. Therefore, we can rewrite the model (13)-(14) in the following more compact form:

$$
\begin{aligned}
x(k+1) & =f(x(k), \theta)+w(k) \\
z(k) & =g(x(k), \theta)+v(k)
\end{aligned}
$$

where $x(k)$ is the vector of state variables at the time point $k, f(.,$.$) is a nonlinear function with \theta$ being a parameter vector to be identified. $w(k)$ and $v(k)$ denote the zero-mean uncorrelated Gaussian noises with covariance matrices $Q_{k}$ and $R_{k}$, respectively. $z(k)$ is the measurement data from experiments at the time point $k$.

It is clear from (15)-(16) that what we need to do is to identify the parameter vector $\theta$ for the purpose of establishing the lateral flow immunoassay model. In the Ref. [45], the EKF method is used to estimate the parameters of the model (15)(16) from the possibly small number of the measured data. It has been shown in [45] that the EKF method is able to jointly estimate the parameters and states simultaneously. However, in practice, the concentration of the materials, the association and dissociation rate constants should all be positive and such a state constraint has not been taken into account in [45]. Therefore, the main aim of this paper is to jointly estimate the parameters and states of the model (15)-(16) with state constraints via the hybrid EKF and switching PSO method from the possibly small number of the measured data.

\section{EXtended KALMAN FiLtering With INEQUALITY CONSTRAINTS}

\section{A. Traditional Extended Kalman Filtering}

In this section, for the convenience of the readers, we first introduce the EKF approach to parameter identification, see e.g. [4], [15], [41] for more details.

The Kalman filter is the optimum state estimator for a linear system. If the system is nonlinear, we may use a linearization process at every time step to approximate the nonlinear system with a linear time-varying (LTV) system. This LTV system is then used in the Kalman filter, resulting in an EKF on the true nonlinear system. Note that although EKF is not necessarily optimal, it often works very well. Discussions on the convergence of EKF can be found in [9], [13] and the references therein.

Consider the following nonlinear system

$$
\begin{aligned}
x(k+1) & =f(x(k))+w(k) \\
y(k) & =g(x(k))+v(k)
\end{aligned}
$$

where $k$ is a non-negative integer, $x(k) \in \mathbb{R}^{n}$ is the system state vector, $y(k) \in \mathbb{R}^{r}$ is the observation vector, and $w(k)$ and $v(k)$ are the system noise and the measurement noise, respectively. $w(k)$ and $v(k)$ are zero-mean white Gaussian stochastic sequences with covariance matrices $Q_{k}$ and $R_{k}$, 
respectively. Here, $f: \mathbb{R}^{n} \rightarrow \mathbb{R}^{n}$ is a nonlinear state transition function and $g: \mathbb{R}^{n} \rightarrow \mathbb{R}^{r}$ is a nonlinear measurement function.

The EKF is implemented by the following consecutive steps:

1) Consider the last filtered state estimate $\hat{x}(k \mid k)$

2) Linearize the system dynamics (17) around $\hat{x}(k \mid k)$

3) Apply the prediction step of the Kalman filter to the linearized system dynamics just obtained, yielding $\hat{x}(k+$ $1 \mid k)$ and $P(k+1 \mid k)$, which are priori state estimate and error covariance at step $k+1$ given knowledge of the process prior to step $k+1$, respectively

4) Linearize the observation equation (18) around $\hat{x}(k \mid k)$

5) Apply the filtering or update cycle of the Kalman filter to the linearized observation dynamics, yielding $\hat{x}(k+$ $1 \mid k+1)$ and $P(k+1 \mid k+1)$

Let

$$
\begin{aligned}
\hat{A}(k) & =\left.\frac{\partial f(x(k))}{\partial x(k)}\right|_{x(k)=\hat{x}(k \mid k)} \\
\hat{C}(k) & =\left.\frac{\partial g(x(k))}{\partial x(k)}\right|_{x(k)=\hat{x}(k \mid k-1)}
\end{aligned}
$$

Assume that $x(0) \sim \mathcal{N}\left(x_{0}, P_{x_{0}}\right), w(k) \sim \mathcal{N}\left(0, Q_{k}\right), v(k) \sim$ $\mathcal{N}\left(0, R_{k}\right)$ with $R_{k}>0$, and that $\{w(k)\}$ and $\{v(k)\}$ are white noise processes uncorrelated with $x(0)$ and with each other. Then, the EKF algorithm can be stated below:

\section{Initialization}

For $k=0$, set

$$
\begin{aligned}
& \hat{x}(0 \mid 0)=E[x(0)]=x_{0}, \\
& P(0 \mid 0)=E\left[\left(x(0)-x_{0}\right)\left(x(0)-x_{0}\right)^{T}\right]=P_{x_{0}} .
\end{aligned}
$$

For $k=1,2,3, \ldots$ compute

\section{Time update ('Predict')}

State estimate time update: $\hat{x}(k \mid k-1)=f(\hat{x}(k-1 \mid k-1))$

Error covariance time update: $P(k \mid k-1)=\hat{A}(k-1) P(k-$ $1 \mid k-1) \hat{A}(k-1)^{T}+Q(k-1)$

\section{Measurement update ('Correct')}

Compute the Kalman gain matrix: $K_{k}=P(k \mid k-$ 1) $\hat{C}(k)^{T}\left[\hat{C}(k) P(k \mid k-1) \hat{C}(k)^{T}+R(k)\right]^{-1}$

Update the estimate with measurement $y(k): \hat{x}(k \mid k)=$ $\hat{x}(k \mid k-1)+K_{k}[y(k)-g(\hat{x}(k \mid k-1))]$

Error covariance measurement update: $P(k \mid k)=(I-$ $\left.K_{k} \hat{C}(k)\right) P(k \mid k-1)$.

In addition, in order to improve the precision of state estimation and also reduce the possible biases, there is a need to properly quantify the parameters $Q$ and $R$ in the
EKF algorithm. To tackle this issue, we use the innovationbased adaptive estimation approach [21], where the covariance matrices $Q(k)$ and $R(k)$ are estimated and then updated iteratively according to the following equations:

$$
\begin{aligned}
R(k) & =C_{v k}+\hat{C}(k) P(k \mid k) \hat{C}(k)^{T} \\
Q(k) & =K_{k} C_{v k} K_{k}^{T}
\end{aligned}
$$

where $C_{v k}$ is the innovation covariance matrix computed through averaging the innovation sequence $s(k)$ inside a moving estimation window of size $N$ as follows

$$
\begin{aligned}
C_{v k} & =\frac{1}{N} \sum_{i=k-N+1}^{k} s(k) s(k)^{T} \\
s(k) & =y(k)-g(\hat{x}(k \mid k-1)) .
\end{aligned}
$$

Based on the above equations, the appropriate values of $Q$ and $R$ can be determined at each iteration.

Remark 1: EKF has proven to be a very practical method in the state estimation of nonlinear systems. Augmenting the unknown parameters to the state vector makes it possible to use EKF for parameter identification too.

\section{B. The Maximum Probability Method}

The EKF algorithm has been successfully used to estimate the state variables of a dynamic system [4], [33], [41] without particular constraints. However, in many engineering practice, there does exist certain restrictions on the system states such as the positivity constraint. A typical example is the lateral flow immunoassay model where the concentration of the materials, the association and dissociation rate constants should all be positive in the real world. Within the recursive framework of traditional EKF, it is fundamentally difficult to incorporate the constraints (algebraic equality and/or inequality) directly on the system states since this is basically a constrained estimation problem. On the other hand, the maximum probability method [29], [30] is well known to be capable of converting the constrained estimation problem to constrained optimization problem after each time step of the EKF algorithm and, therefore, this method is chosen to handle the constrained EKF problem in this paper with applications in state and parameter estimation for lateral flow immunoassay systems.

Consider the nonlinear dynamical system of (17)-(18) with the following additional constraint

$$
D x \leq d
$$

where $D$ is a known $s \times n$ constant matrix with full row rank, $s$ is the number of constraints, $n$ is the number of state variables 
and $s \leq n$. (25) means that each entry of the vector $D x-d$ is non-positive. It is known from [2], [29], [30] that, based on Kalman filtering theory, the state estimate of $x$ maximizes the conditional probability density function:

$$
\mathcal{P}(x \mid Y)=(2 \pi)^{-\frac{n}{2}}|P|^{-\frac{1}{2}} \exp \left\{-\frac{1}{2}(x-\bar{x})^{T} P^{-1}(x-\bar{x})\right\}
$$

where $n$ is the dimension of $x, P$ is the covariance of the Kalman filter estimate, $Y$ denotes the column vector that contains the measurements $\left\{y_{0}, y_{1}, \ldots, y_{k}\right\}$, and $\bar{x}$ is the conditional mean of $x$ given the measurements $Y$.

The constrained EKF can be derived by finding an estimate $\tilde{x}$ such that the conditional probability $\mathcal{P}(\tilde{x} \mid Y)$ is maximized and $\tilde{x}$ satisfies the constraint (25). Since maximizing $\mathcal{P}(\tilde{x} \mid Y)$ is equivalent to maximizing its natural logarithm, the problem to be solved can be expressed as

$$
\begin{array}{r}
\max \ln \mathcal{P}(\tilde{x} \mid Y) \Longrightarrow \min (\tilde{x}-\bar{x})^{T} P^{-1}(\tilde{x}-\bar{x}) \\
\text { such that } D \tilde{x} \leq d
\end{array}
$$

So far, it can be seen that the constrained state estimation problem has been converted into an equivalent constrained optimization problem that can be solved after each time step of the EKF algorithm.

\section{Switching Particle SWARm Optimization For CONSTRAINED OPTIMIZATION PROBLEMS}

\section{A. Switching Particle Swarm Optimization Algorithm}

PSO is a popular stochastic optimization algorithm proposed by Kennedy and Eberhart in 1995 [12]. The main idea of PSO algorithm was based on the simulation of simplified social models such as bird flocking and fish schooling. In PSO [35], a swarm consists of $S$ particles moving around in a $D$ dimensional search space. The position of the $i$ th particle is denoted by a vector, $x_{i}(k)=\left(x_{i 1}(k), x_{i 2}(k), \cdots, x_{i D}(k)\right)$, where $x_{i n}(k) \in\left[x_{\min , n}, x_{\max , n}\right](1 \leq n \leq D)$ with $x_{\min , n}$ and $x_{\max , n}$ being lower and upper bounds for the $n$th dimension, respectively. During the search process, the particle successively adjusts its position towards the global optimum according to the two factors: the best position encountered by itself ( pbest) denoted as $p_{i}=\left(p_{i 1}, p_{i 2}, \cdots, p_{i D}\right)$ and the best position in the whole swarm (gbest) denoted as $p_{g}=\left(p_{g 1}, p_{g 2}, \cdots, p_{g D}\right)$. The velocity of the $i$ th particle at the $k$ th iteration is represented by $v_{i}(k)=$ $\left(v_{i 1}(k), v_{i 2}(k), \cdots, v_{i D}(k)\right)$, and is limited to a maximum velocity $v_{i, \max }=\left(v_{i \max , 1}, v_{i \max , 2}, \cdots, v_{i \max , D}\right) \cdot r_{1, j}$ and $r_{2, j}$ are two uniform random number samples from $U(0,1)$. The parameters $c_{1}$ and $c_{2}$ are called acceleration coefficients, namely, cognitive and social parameters, respectively. The velocity and position of the particle at next iteration are updated according to the following equations:

$$
\begin{array}{ll}
v_{i}(k+1)= & w v_{i}(k)+c_{1} r_{1}\left(p_{i}(k)-x_{i}(k)\right) \\
& +c_{2} r_{2}\left(p_{g}(k)-x_{i}(k)\right), \\
x_{i}(k+1)= & x_{i}(k)+v_{i}(k+1),
\end{array}
$$

where $w$ is the inertia weight. It is shown that a larger inertia weight tends to facilitate the global exploration and a smaller inertia weight achieves the local exploration to fine-tune the current search area [28].

In this paper, a modified version of the traditional PSO algorithm, namely, switching PSO algorithm [35], is employed to tackle the constrained optimization problem. The basic difference between the traditional PSO and the switching PSO algorithms is that, the contradiction between the local search and global search in the traditional PSO algorithm is removed in the switching PSO algorithm. Generally speaking, in the early search stage, the particle in the swarm should keep its independence and swarm's diversity, which helps to enlarge the search scope and avoid premature problem happening. In the latter stage of the search process, all the swarms may converge to the best particle for getting more accurate solution. In the switching PSO algorithm, a mode-dependent velocity updating equation with Markovian switching parameters is introduced to overcome the contradiction between the local search and global search [35]. The velocity and position of the particle at next iteration are updated with Markovian jumping parameters according to the following equations:

$$
\begin{array}{ll}
v_{i}(k+1)= & w(\xi(k)) v_{i}(k)+c_{1}(\xi(k)) r_{1}\left(p_{i}(k)-x_{i}(k)\right) \\
& +c_{2}(\xi(k)) r_{2}\left(p_{g}(k)-x_{i}(k)\right), \\
x_{i}(k+1)= & x_{i}(k)+v_{i}(k+1),
\end{array}
$$

where $w(\xi(k)), c_{1}(\xi(k))$ and $c_{2}(\xi(k))$ are the inertia weight and acceleration coefficients. All of them are mode-dependent on a non-homogeneous Markov chain $\xi(k)(k \geq 0)$, which take values in a finite state space $\mathcal{S}=\{1,2, \cdots, N\}$ with probability transition matrix $\Pi^{(k)}=\left(\pi_{i j}^{(k)}\right)_{N \times N} \cdot \pi_{i j}^{(k)} \geq 0$ $(i, j \in \mathcal{S})$ is the transition rate from $i$ to $j$ and $\sum_{j=1}^{N} \pi_{i j}^{(k)}=1$. Usually, in the initiative stage, the inertia weights $w(\xi(k))$ and $c_{1}(\xi(k))$ are larger than those of in the latter stage for maintaining the swarm diverse, while $c_{2}(\xi(k))$ is compara- 
tively smaller than that in the early stage for getting the global optimum more accurately.

In order to maintain a balance between global search and local search, $\Pi^{(k)}$ should be adjusted by the current search information such as the swarm diversity and the current best solution in the swarm. The diversity of a swarm is used to describe the distribution of the each individual particle in a whole field, which can be calculated by the diversity-measure [35], [36] as follows:

$$
\operatorname{Div}(k)=\frac{1}{S \cdot|L|} \sum_{i=1}^{S} \sqrt{\sum_{d=1}^{D}\left(x_{i d}(k)-\bar{x}_{d}(k)\right)^{2}}
$$

where $S$ is the swarm size, $|L|$ is the length of the longest diagonal in the search space, $D$ is the dimension of the objective problem, $x_{i d}$ is the $d$ th value of the $i$ th particle, while $\bar{x}_{d}$ is the $d$ th value of the average point $\bar{x}$ in the whole swarm that can be computed by:

$$
\bar{x}_{d}(k)=\frac{1}{S} \sum_{i=1}^{S} x_{i d}(k) .
$$

Let $h$ be a coefficient to be used to differentiate the early search stage and late search stage. Here, $h$ is chosen as $h \in$ $\left[\frac{2}{3}, \frac{4}{5}\right] . T_{\max }$ is the largest iterations for the algorithm. For the sake of simplicity, we set $N=2$. The pseudo code of SPSO algorithm is described as follows by above discussion [35]:

Initialize the velocity $v_{i}$ and position $x_{i}(k=0)$.

Set the $w(\xi(0)), c_{1}(\xi(0)), c_{2}(\xi(0)), \Pi^{(0)}$ and initial mode $s=1$.

while (not satisfying the termination condition)

do

for $i=1$ to particle numbers $S$; \{

Evaluate fitness of every particle;

Update the swarm best solution $p_{g}$ and the particle best solution $p_{i}$;

if $\left(0<k<h * T_{\max }\right)$ and diversity $\operatorname{Div}(k)<$ a set value $[0.3,0.7])$

increase the $\chi$ of $\Pi^{(k)}=\left(\begin{array}{ll}\chi & 1-\chi \\ \chi & 1-\chi\end{array}\right)(\chi \in$

end;

if $\left(k>h * T_{\max }\right)$ and $p_{g}<$ a set value

$$
\Pi^{(k)}=\left(\begin{array}{cc}
\rho & 1-\rho \\
\rho & 1-\rho
\end{array}\right)(\rho \in[0.05,0.2]) ;
$$

end;

Calculate particle new velocity $v_{i}(k+1)$ by Eq.(29);
Calculate particle new position $x_{i}(k+1)$ by Eq.(29);

$\mathrm{k}=\mathrm{k}+1$;

\}

end do;

end;

\section{B. The Penalty Function Approach}

PSO as a global evolutionary algorithm has been successfully applied in a variety of areas especially for unconstrained optimization problems [10], [12], [20], [27], [28], [35], [37]. Although PSO has been developed primarily as the unconstrained optimization method, it also performs well when used in constrained optimization problems [22] that occur frequently in the real world. The penalty function approach has been the most popular constraint-handling technique due to its simple principle. By using the penalty function approach, a constrained optimization problem can be converted into a corresponding unconstrained optimization one by adding a penalty term to the objective function [22], [32].

In this paper, a non-stationary multi-stage assignment penalty function [22] is used to cope with the constrained optimization. The penalty function is defined as

$$
F(x)=f(x)+h(k) H(x), \quad x \subset \mathbb{R}^{n}
$$

where $f(x)$ is the original objective function of the constrained optimization problem in Eq. (25); $h(k)$ is a dynamically modified penalty value with $k$ being the algorithm's current iteration number; and $H(x)$ is a penalty factor defined as

$$
H(x)=\sum_{i=1}^{s} \theta\left(q_{i}(x)\right) q_{i}(x)^{\gamma\left(q_{i}(x)\right)}
$$

where $q_{i}(x)=\max \left\{0, g_{i}(x)\right\} \quad(i=1, \ldots, s)$, the function $q_{i}(x)$ is a relative violated function of the constraints, $s$ is the number of the constrains, $\theta\left(q_{i}(x)\right)$ is a multi-stage assignment function, $\gamma\left(q_{i}(x)\right)$ is the power of the penalty function, and $g_{i}(x)=D x-d$ are the constraints described in Eq. (25).

Finally, the flowchart of our purposed hybrid EKF and Switching PSO algorithm is presented in Fig. 2

\section{Simulation Results ANd Discussion}

In this section, the proposed novel hybrid EKF and switching PSO method is utilized to joint state and parameter estimation of the sandwich-type lateral flow immunoassay model via the short time series data used in the Ref. [45]. 


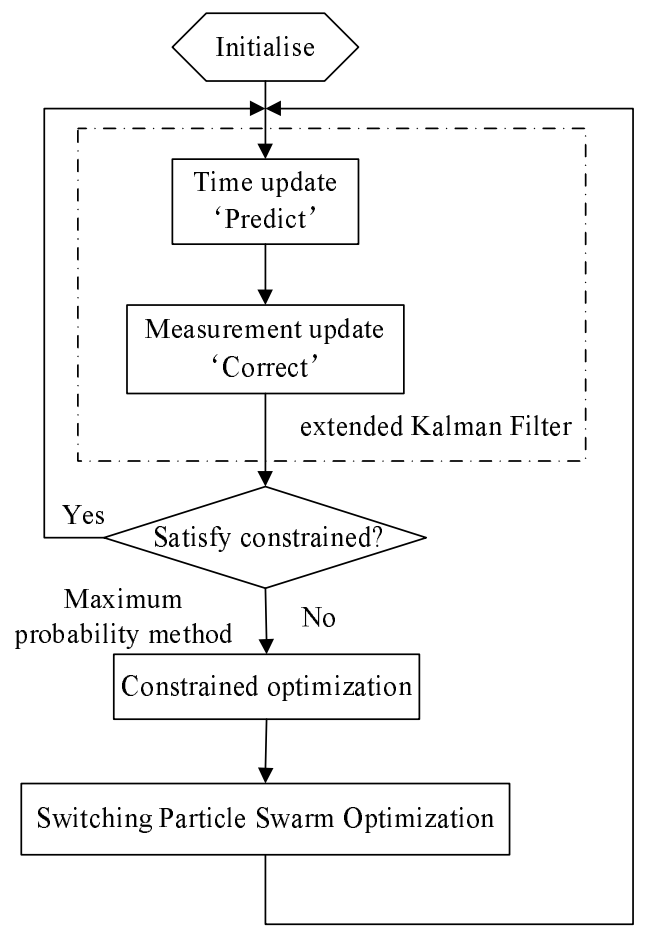

Fig. 2. The flowchart of the hybrid EKF and Switching PSO algorithm.

Take

$x_{0}=[5,6.5,0,13,0,0]^{T}$,

$k_{0}=[0.03,0.0001,0.01,0.0001,0.04,0.0001,0.04,0.0001,2.2]^{T}$ as the initial values of the state variables and parameters, respectively. Regarding the penalty function parameters, we use the same values as used in [22], [44]. Specifically, if $q_{i}(x)<1$, then $\gamma\left(q_{i}(x)\right)=1$, otherwise $\gamma\left(q_{i}(x)\right)=2$. Moreover, if $q_{i}(x)<0.001$, then $\theta\left(q_{i}(x)\right)=10$; else, if $q_{i}(x) \leq 0.1$, then $\theta\left(q_{i}(x)\right)=20$; else, if $q_{i}(x) \leq 1$, then $\theta\left(q_{i}(x)\right)=100$; otherwise $\theta\left(q_{i}(x)\right)=300$. The function $h($. is set as $h(k)=\sqrt{k}$. Both the identified state variables and parameters are shown in Fig. 3 and Fig. 4, which are depicted in the form of time series. The time series for error covariances is also obtained, simultaneously, which are plotted in Fig. 5 and Fig. 6. Fig. 7 shows the time series for the noise variance $Q_{k}$ and $R_{k}$.

It is observed from Fig. 3 and Fig. 4 that the simulated results satisfy the constraints on the state variables, i.e., the concentration of the materials, the association and dissociation rate constants should be positive. Also, we can see from Fig. 5 and Fig. 6 that the estimation covariances are small, which means that our model fits the data very well.

Furthermore, to evaluate the model quality in a quantitative

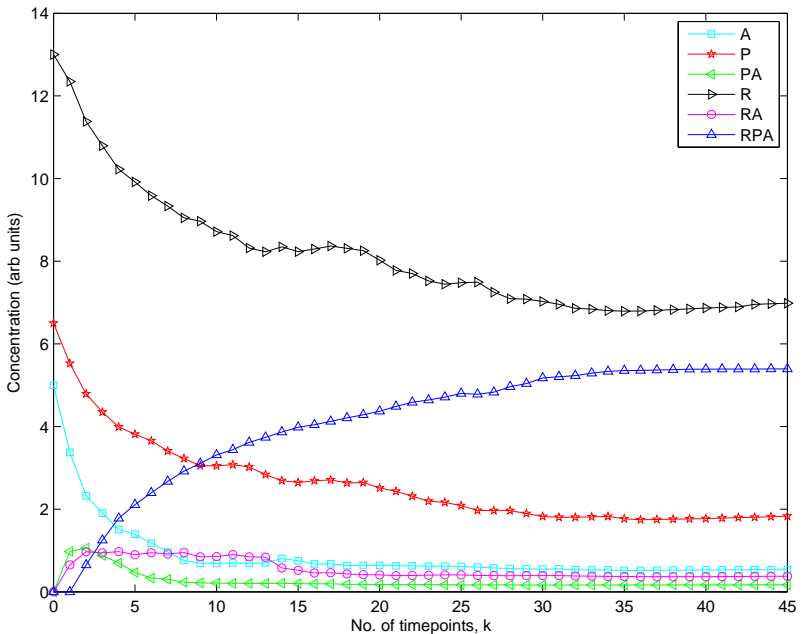

Fig. 3. The estimated time series of states $A, P, P A, R, R A, R P A$.

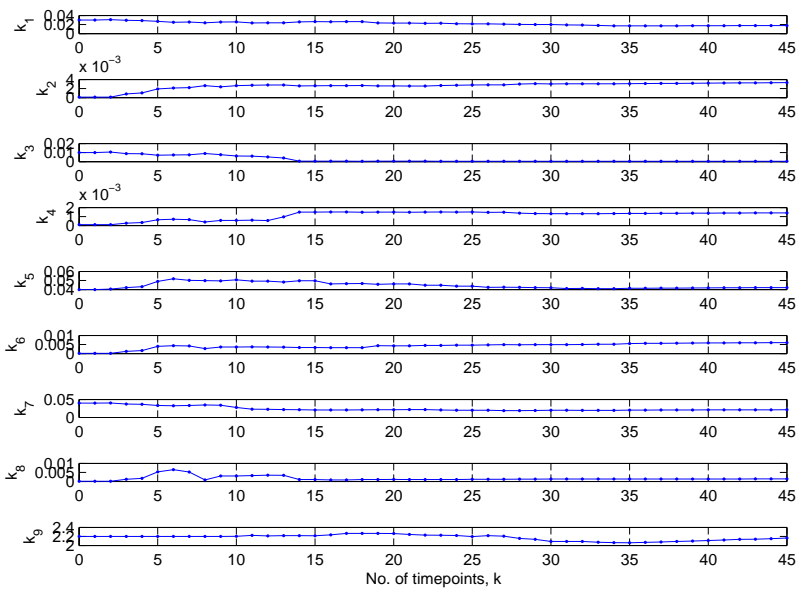

Fig. 4. The estimated time series of parameters $k_{1}, k_{2}, k_{3}, k_{4}, k_{5}$, $k_{6}, k_{7}, k_{8}, k_{9}$.

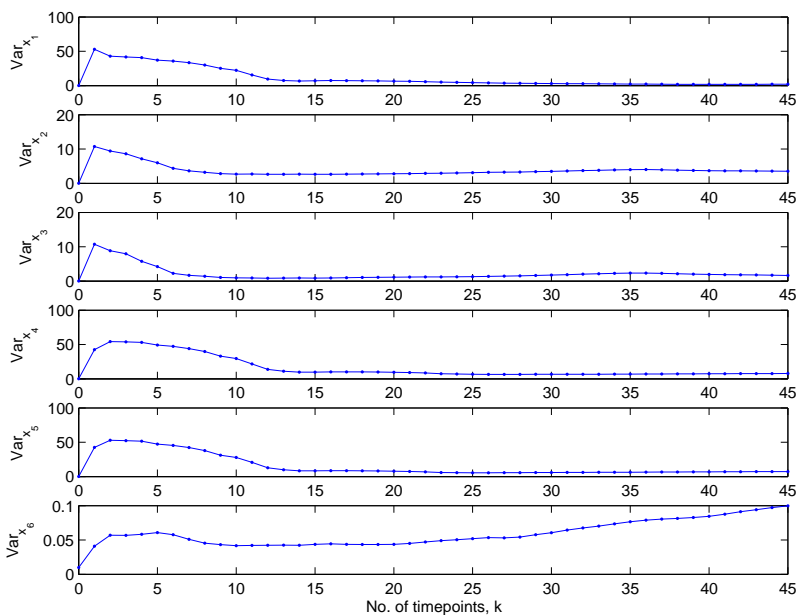

Fig. 5. The variances estimated time series of states $A, P, P A, R$, $R A, R P A$ 


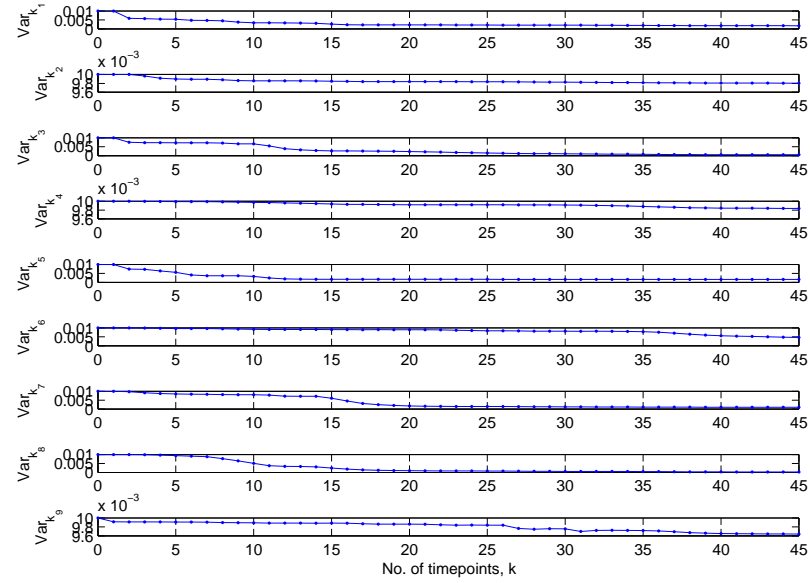

Fig. 6. The variances of estimated time series of parameters $k_{1}, k_{2}$, $k_{3}, k_{4}, k_{5}, k_{6}, k_{7}, k_{8}, k_{9}$.

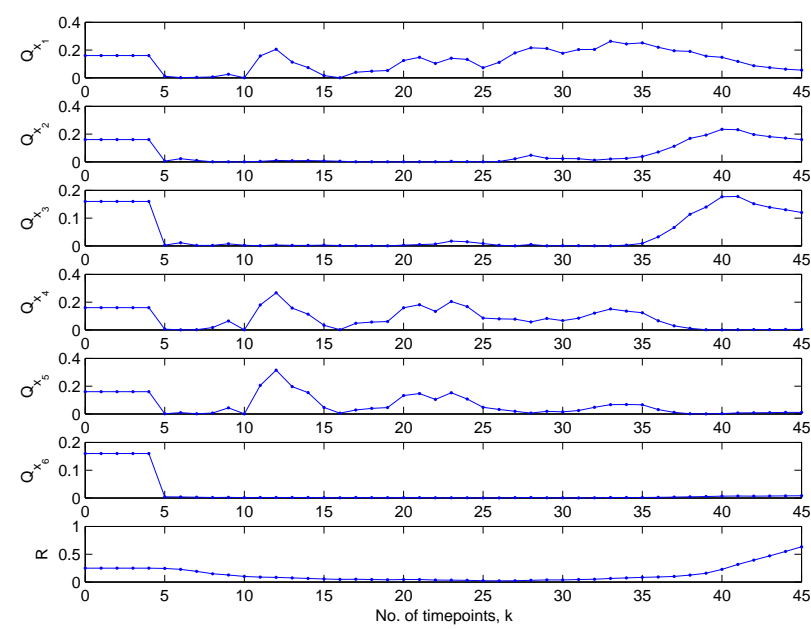

Fig. 7. The noise variances estimated time series of $Q_{k}$ and $R_{k}$.

way, let us introduce the following criterion for the modeling errors (error ratio in percentage) between the actual and the model predicted data [19], [42]:

$$
\text { Error ratio }=100 \times \frac{1}{l} \sum_{c=1}^{l}\left[\sqrt{\frac{\sum_{k=1}^{s}\left(y_{c k}-\hat{y}_{c k}\right)^{2}}{\sum_{k=1}^{s}\left(y_{c k}\right)^{2}}}\right] \%
$$

where $l$ is the number of observations (dimension) involved in the modeling ( $l=1$ in this paper); $s$ is the number of observations (length), and $y_{c k}$ is the actual value for $c$ th observation at the $k$ th time point. The results are given in Table I. It can be concluded that the model identified by the proposed algorithm does make more practical sense than the unconstrained EKF. It is noticed that the error ratio of the proposed algorithm is bigger than the unconstrained EKF through only 45 time points (images), which is mainly due to the positivity constraints on the system states.
TABLE I

QUANTITATIVE MODEL EVALUATION FOR TIME SERIES WITH DIFFERENT CONCENTRATIONS

\begin{tabular}{|c|c|c|}
\hline \hline Experiment & Unconstrained & Constrained \\
\hline Error ratio & $1.05 \%$ & $3.26 \%$ \\
\hline \hline
\end{tabular}

\section{CONCLUSiOnS}

In this paper, we have presented a hybrid EKF and switching PSO algorithm for incorporating inequality constraints on the system states. Note that such constraints are often ignored within the framework of extended Kalman filtering theory. By using the maximization probability method, the constraints on state variables in dynamic state estimation problem can be handled by combining the EKF algorithm with a constrained optimization problem. Then, the constrained optimization problem has been solved by a recently proposed switching PSO algorithm together with the penalty function method. In the end, the proposed algorithm has been successfully applied to identify the constrained parameters and states of the lateral flow immunoassay model simultaneously. The simulation results has demonstrated the effectiveness of the proposed method.

\section{ACKNOWLEDGMENTS}

This work was supported in part by the International Science and Technology Cooperation Project of China under Grant 2009DFA32050, Natural Science Foundation of China under Grants 61104041, International Science and Technology Cooperation Project of Fujian Province of China under Grant 200910016

\section{REFERENCES}

[1] C. An, T. Yoshiki, G. Lee, Y. Okada, Evaluation of a rapid qualitative prostate specific antigen assay, the One Step PSA ${ }^{T M}$ test, Cancer Lett, vol. 162, no. 2, pp. 135-139, 2001.

[2] B. D. O. Anderson and J. B. Moore, Optimal filtering, Prentice Hall, 1979.

[3] http://commons.wikimedia.org/wiki/File:Lateral_Flow_Assay.jpg

[4] A. Corigliano and S. Mariani, Parameter identification in explicit structural dynamics: performance of the extended Kalman filter, Computer Methods in Applied Mechanics and Engineering, vol. 193, pp. 38073835, 2004.

[5] M. Du, Z. Fang and H. Fei, Application of photoelectric sensor to quantitative determination of immunochro-matographic assay strip, Chinese Journal of Scientific Instrument, vol. 36, no. 7, pp. 671-673, 2005.

[6] K. Faulstich, R. Gruler, M. Eberhard and K. Haberstroh, Developing rapid mobile POC systems. Part 1:Devices and applications for lateralflow immunodiagnostics, IVD Technology, vol. 13, no. 6, pp. 47-53, 2007. 
[7] J. Gantelius, C. Hamsten, M. Neiman, J. M. Schwenk, A. Persson, H. Andersson-Svahn, A lateral flow protein microarray for rapid determination of contagious bovine pleuropneumonia status in bovine serum, Journal of Microbiol Methods, vol. 82, no. 1, pp. 11-18, 2010.

[8] L. Huang, Y. Zhang, C. Xie, J. Qu, H. Huang and X. Wang, Research of reflectance photometer based on optical absorption, International Journal for Light and Electron Optics, vol. 121, no. 19, pp. 1725-1728, 2010.

[9] S. Huang and D. Dissanayake, Convergence and consistency analysis for extended Kalman filter based SLAM, IEEE Trans. Robotics, vol. 23, no. 5, pp. 1036-1049, 2007.

[10] V. Kadirkamanathan, K. Selvarajah and P. Fleming, Stability analysis of the particle dynamics in particle swarm optimizer, IEEE Trans Evol. Comput., vol. 10, no. 3, pp. 245-255, 2006.

[11] J. Kaur, K. Singh, R. Boro, K. Thampi, M. Raje and G. Varshney, Immunochromatographic dipstick assay format using gold nanoparticles labeled protein-hapten conjugate for the detection of atrazine, Environmental Science and Technology, vol. 41, no. 14, pp. 5028-5036, 2007.

[12] J. Kennedy and R. Eberhart, Particle swarm optimization, Proc. IEEE International Conference On Neural Network, pp. 1942-1948, 1995.

[13] A.J. Krener, The convergence of the extended Kalman filter, IEEE Directions in Mathematical Systems Theory and Optimization, pp. 173182, 2003.

[14] L. Lang, W. Chen, BR. Bakshi, P. K. Goel and S. Ungarala, Bayesian estimation via sequential Monte Carlo sampling-Constrained dynamic systems, Automatica, vol. 3, pp. 1615-1622, 2007.

[15] F. Lewis, L. Xie and D. Popa, Optimal and robust estimation, CRC press, 2007.

[16] D. Li, S. Wei, H. Yang, Y. Li and A. Deng, A sensitive immunochromatographic assay using colloidal gold-antibody probe for rapid detection of pharmaceutical indomethacin in water samples, Biosensors and Bioelectronics, vol. 24, no. 7, pp. 2277-2280, 2009.

[17] J. Li, A. Ouellette, L. Giovangrandi, D. Cooper, A. Ricco and G. Kovacs, Optical scanner for immunoassays with up-converting phosphorescent labels, IEEE Trans. Biomedical Engineering, vol. 55, no. 5, pp. 15601571,2008

[18] Y. Li, N. Zeng and M. Du, Study on the methodology of quantitative gold immunochromatographic strip assay, Proc. 2010 International Workshop on Intelligent Systems and Application, pp. 182-185, 2010.

[19] L. Ljung, System Identification: Theory for the User, 2nd ed. Upper Saddle River, NJ: Prentice-Hall, 1999.

[20] R. Mendes, J. Kennedy and J. Neves, The fully informed particle swarm: Simpler, maybe better, IEEE Trans Evol. Comput., vol. 8, no. 3, pp. 204210, 2004

[21] A. Mohamed, K. Schwarz, Adaptive Kalman filtering for INS/GPS, Journal of Geodesy, vol. 73, no. 4, pp. 193-203, 1999.

[22] K. Parsopoulos and M. Vrahatis, Particle swarm optimization method for constrained optimization problems, Proceedings of the EuroInternational Symposium on Computational Intelligence, pp. 214-220, 2002.

[23] S. Qian and H. Haim, A mathematical model of lateral flow bioreactions applied to sandwich assays, Analytical Biochemistry, vol. 322, no. 1, pp. 89-98, 2003.

[24] S. Qian and H. Haim, Analysis of lateral flow biodetectors: competitive format, Analytical Biochemistry, vol. 326, no. 2, pp. 211-224, 2004.

[25] M. Quach, N. Brunel and F. d'Alché-Buc, Estimating parameters and hidden variables in non-linear state-space models based on ODEs for biological networks inference, Bioinformatics, vol. 23, no. 23, pp. 32093216, 2007
[26] C. Raphael and Y. Harley, Lateral flow immunoassay, Humana Press, 2008.

[27] A. Ratnaweera, S. K. Halgamure and H. C. Watson, Self-organizing hierarchical particle swarm optimizer with time-varying acceleration coefficients, IEEE Trans Evol. Comput., vol. 8, pp. 240-255, 2004.

[28] Y. Shi, R C. Eberhart, Empirical study of particle swarm optimization, Proc. 1999 IEEE congress on evolutionary computation, pp. 1945-1950, 1999.

[29] D. Simon and T. Chia, Kalman filtering with state constrains: a survey of linear and nonlinear algorithms, IET Control Theory and Applications, vol. 4, no. 8, pp. 1303-1318, 2010.

[30] D. Simon and D. L. Simon, Aircraft turbofan engine health estimation using constrained Kalman filtering, ASME Journal of Engineering for Gas Turbines and Power, vol. 127, no. 2, pp. 323-328, 2005.

[31] N. Jamshidi, BØ. Palsson, Formulating genome-scale kinetic models in the post-genome era, Molecular Systems Biology, vol. 4, no. 171, 2008.

[32] C. Sun, J. Zeng and J. Pan, An improved particle swarm optimization with feasibility-based rules for constrained optimization problems, Proc. 22nd International Conference on Industrial, Engineering and Other Applications of Applied Intelligent Systems, pp. 202-211, 2009.

[33] X. Sun, L. Jin and M. Xiong, Extended Kalman filter for estimation of parameters in nonlinear state-space models of biochemical networks, PLoS ONE, vol. 3, no. 11, e3758, 2008

[34] R. Tanaka, T. Yuhi, N. Nagatani, T. Endo, K. Kerman and Y. Takamura, A novel enhancement assay for immunochromatographic test strips using gold nanoparticles, Anal Bioanal Chem, vol. 385, no. 8, pp. 1414-1420, 2006.

[35] Y. Tang, Z. Wang and J. Fang, Parameters identification of unknown delayed genetic regulatory networks by a switching particle swarm optimization algorithm, Expert Systems with Applications, vol. 38, pp. 2523-2535, 2011.

[36] Y. G. Tang and X. Guan, Parameter estimation for time-delay chaotic system by particle swarm optimization, Chaos, Solitons and Fractals, vol. 42, no. 5, pp. 3132-3139, 2009.

[37] Y. Valle, G. Venayagamoorthy, S. Mohagheghi, J. Hernandez and R. Harley, Particle swarm optimization: basic concepts, variants and applications in power systems, IEEE Trans Evol. Comput., vol. 12, no. 2, pp. 171-195, 2008.

[38] Z. Gao, T. Breikin and H. Wang, Reliable observer-based control against sensor failures for systems with time delays in both state and input, IEEE Transactions on Systems Man and Cybernetics, part A, vol. 38, no. 5, pp. 1018-1029, 2008.

[39] Z. Gao, X. Shi, S. Ding, Fuzzy state/disturbance observer design for T-S fuzzy systems with application to sensor fault estimation, IEEE Transactions on Systems Man and Cybernetics, part B, vol. 38, no. 3, pp. 875-880, 2008.

[40] Z. Gao, X. Dai, T. Breikin, H. Wang, Novel parameter identification by using a high-gain observer with application to a gas turbine engine, IEEE Transactions on Industrial Informatics, vol. 4, no. 4, pp. 271-279, 2008.

[41] Z. Wang, X. Liu, Y. Liu, J. Liang and V. Vinciotti, An extended Kalman filtering approach to modeling nonlinear dynamic gene regulatory networks via short gene expression time series, IEEE/ACM Trans. Computional Biology and Bioformatics, vol. 6, no. 3, pp. 410-419, 2009.

[42] Z. Wang, F. Yang, D. W. C. Ho, S. Swift, A. Tucker and X. Liu, Stochastic dynamic modeling of short gene expression time series data, IEEE Trans. NanoBioscience, vol. 7, no. 1, pp. 44-55, 2008.

[43] P. Yager, T. Edwards, E. Fu, K. Helton, K. Nelson, M. R. Tam, 
B. H. Weigl, Microfuidic diagnostic technologies for global public health, Nature vol. 442, pp. 412-418, 2006.

[44] J. Yang, Y. Chen, J. Horng and C. Kao, Applying family competition to evolution strategies for constrained optimization, Lecture Notes in Computer Science, vol. 1213, pp. 201-211, 1997.

[45] N. Zeng, Z. Wang, Y. Li, M. Du and X. Liu, Inference of nonlinear state-space models for sandwich-type lateral flow immunoassay using extended Kalman filtering, IEEE Transactions on Biomedical Engineering, vol. 58, no. 7, pp. 1959-1966, 2011.

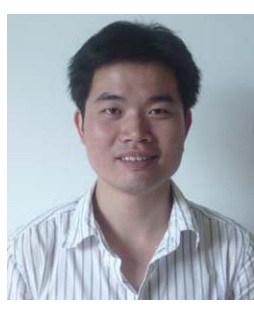

Nianyin Zeng was born in Fujian Province, China, in 1986. He received the B.S. degree in Electrical Engineering and Automation from Fuzhou University, Fuzhou, China, in 2008, where he is currently working toward the Ph.D. degree in Electrical Engineering.

$\mathrm{He}$ is the author or coauthor of several technical papers and also a very active reviewer for many international journals. His current research interests include nonlinear filtering, time-series modeling, and bioinformatics.

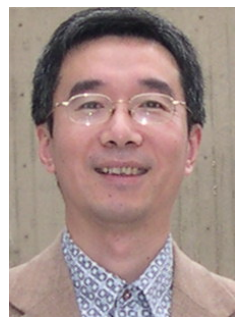

Zidong Wang was born in Jiangsu, China, in 1966. He received his B.Sc. degree in Mathematics in 1986 from Suzhou University, Suzhou, China, the M.Sc. degree in Applied Mathematics in 1990 and the Ph.D. degree in Electrical and Computer Engineering in 1994, both from Nanjing University of Science and Technology, Nanjing, China.

Dr. Wang is now a Professor of Dynamical Systems and Computing at Brunel University in the UK. His research interests include dynamical systems, signal processing, bioinformatics, control theory and applications. He has published more than 200 papers in refereed international journals.

Dr. Wang is currently serving as an Associate Editor for 12 international journals including IEEE Transactions on Automatic Control, IEEE Transactions on Neural Networks, IEEE Transactions on Signal Processing, IEEE Transactions on Systems, Man, and Cybernetics - Part C and IEEE Transactions on Control Systems Technology.

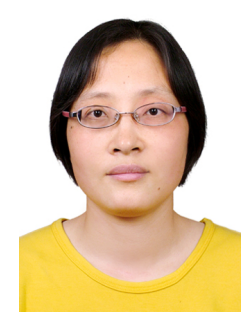

Yurong Li was born in Fujian Province, China, in 1973. She received her Master degree in Industry Automation and Ph.D. in Control Theory and Control Engineering from Zhejiang University, Zhejiang, Chian in 1997 and 2001, respectively.

Now she is an Associate Professor at Fuzhou University. And since 2007, she is the member of Fujian Key Laboratory of Medical Instrumentation \& Pharmaceutical Technology. Her research interests include biomedical instrument and intelligent information processing.

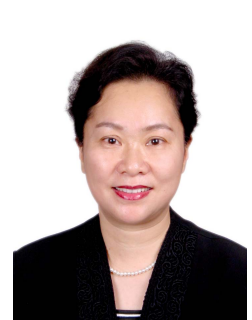

Min Du was born in Fujian Province, China, in 1955. She received her Ph.D. in Electrical Engineering from Fuzhou University, Fuzhou, China, in 2005.

Now she is a professor and doctorial supervisor at Fuzhou University. And since 2007, she is the associate director of Fujian Key Laboratory of Medical Instrumentation \& Pharmaceutical Technology. Her research interests include smart instrument and photoelectrical system.

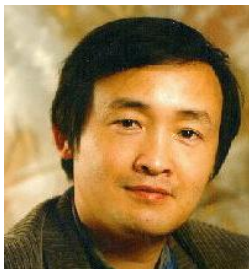

Xiaohui Liu received the B.Eng. degree in computing from Hohai University, Nanjing, China, in 1982 and the Ph.D. degree in computer science from Heriot-Watt University, Edinburg, U.K., in 1988.

$\mathrm{He}$ is currently a Professor of Computing at Brunel University. He leads the Intelligent Data Analysis (IDA) Group, performing interdisciplinary research involving artificial intelligence, dynamic systems, image and signal processing, and statistics, particularly for applications in biology, engineering and medicine. Professor Liu serves on editorial boards of four computing journals, founded the biennial international conference series on IDA in 1995, and has given numerous invited talks in bioinformatics, data mining and statistics conferences. 